\title{
COREA DEL SUR: UNA MIRADA A LO ACONTECIDO EN ECONOMÍA Y RELACIONES INTERCOREANAS DURANTE 2018
}

\author{
Ángel Licona Michel \\ Universidad de Colima
}

\section{RESUMEN}

La República de Corea (Corea del Sur) es uno de los países que se conocen por su superación económica, la cual logró a partir de los años sesenta por medio de avances tecnológicos. Asimismo, ha presentado con el tiempo altas de crecimiento en su Producto Interno Bruto (PIB) que hacen de su economía un actor importante por la dinámica de sus productos que dinamiza las exportaciones y las importaciones, lo que convierten al país en un competidor importante en la escena internacional.

Pero Corea del Sur no sólo se conoce por sus logros económicos y tecnológicos, sino también porque comparte con la República Popular Democrática de Corea (o Corea del Norte), desde 1948, un territorio. En este contexto, además de la parte económica y de las relaciones intercoreanas que a lo largo de la historia han mostrado diferencias y tensiones de tipo político y militar, es un hecho la intromisión de naciones como Estados Unidos de Norteamérica (EUA), la República Popular China (RPCh), Rusia y Japón, entre otras, quienes consideran que se pueden versafectados sus intereses en la región.

En este capítulo se hace un análisis de la economía y las relaciones intercoreanas en 2018. 


\section{INTRODUCCIÓN}

Corea del Sur es un país que se ha caracterizado por su esfuerzo para transformar sus procesos de producción. Siempre ha tenido que superar obstáculos, primero por ser colonia de Japón hasta 1945, después por la división del país en Corea del Sur y Corea del Norte en 1948. Con la división del país la situación económica y social no le fue favorable, además tenían que estar compitiendo con naciones como Japón y la RPCh, entre otras de la región y del mundo.

Las vicisitudes que se han vivido en Corea del Sur han contribuido para que actores políticos, económicos, tecnológicos y sociales tracen el camino a seguir para mejorar la situación económica de las empresas, al igual que de las personas, así como el trabajo que deberán emprender en la esfera política nacional con los países vecinos y en el contexto internacional, para atender las complicadas relaciones intercoreanas.

El trabajo realizado en las partes económica y política muestra resultados fructíferos a la población de Corea del Sur, que en el año 2018 alcanzará tasas de crecimiento de 3\% en su PIB; asimismo, el ingreso per cápita superará los 30 mil dólares anuales. Aunado a su dinámica económica, presentan un crecimiento en sus exportaciones en el que sobresalen productos relacionados con petrolíferos, semiconductores, embarcaciones y automotores. De igual manera, en la esfera política ha disminuido la tensión militar en la península coreana, con lo que se logró conformar entre los líderes de las dos Coreas la Tercera Cumbre Intercoreana, y con ello las condiciones para que pudiera presentarse una agenda de diálogo entre los gobiernos de EUA y Corea del Norte. Asimismo, se concretará la primera reunión entre un presidente estadounidense y un líder norcoreano.

Es así como en este artículo se reflexiona acerca de los aspectos económicos y las relaciones intercoreanas que, en nuestra opinión marcan un hito en la historia de los acontecimientos más importantes de Corea del Sur durante 2018. Para poder reflexionar al respecto fue necesario recurrir a datos económicos y a algunos episodios de tensión militar entre las dos Coreas que se suscitaron en fechas anteriores a 2018. 


\section{ECONOMÍA}

Corea del Sur continúa teniendo un crecimiento económico acelerado desde la década de los sesenta; por ejemplo, de acuerdo con datos del Banco Mundial (BM, 2018), de 1961 a 2017 se tuvo en promedio un crecimiento de $7.4 \%$ en el PIB. Cálculos de la Organización para la Cooperación y el Desarrollo Económico (OECD, por sus siglas en inglés) y el Banco de Corea (BOK, por sus siglas en inglés) indican que durante 2018 el crecimiento del PIB será de $3 \%$, esto por el ritmo de sus exportaciones y por la política fiscal (OECD, 2018; BOK, 2018, y Yonhap, 2018). Dichos cálculos reflejan el trabajo desarrollado en el país, que durante el primer trimestre de 2018 alcanzó un crecimiento de 1.1\% en el PIB, lo cual posicionó a Corea del Sur como el quinto país de la OECD con mayores tasas de crecimiento, mientras el promedio de crecimiento en el PIB de países miembros de la OECD fue de $0.5 \%$ (Lee, 2018).

El promedio de crecimiento en el PIB contribuye a la riqueza de la sociedad surcoreana; por ejemplo, el ingreso per cápita en 1961 era de 94 dólares, para 1971 de 301 dólares, en 1981 de 1870 dólares, en 1991 se llegó a 7523 dólares, en 2001 alcanzó los 11253 dólares, en 2011 los 24080 dólares y en 2017 los 29743 dólares (BM, 2018); en 2018 se obtendrán arriba de 30 mil dólares anuales, ello por la dinámica que presenta la economía surcoreana y que muchos países de América como México, Brasil, Chile y Argentina, entre otros, no han podido alcanzar en lo que va del siglo XXI (BM, 2018).

Para Corea del Sur, mantener el ritmo de crecimiento en el PIB e incrementar la riqueza de la sociedad no ha sido tarea sencilla; por ejemplo, en 2017, por la decisión del gobierno surcoreano de instalar en su territorio un sistema antimisiles procedente de EUA, para frenar las amenazas del gobierno de Corea del Norte, se generó descontento en la región y se deterioraron las relaciones diplomáticas y económicas con la RPCh, que es su principal socio comercial. La RPCh, como represalia por la decisión del gobierno surcoreano, prohibió a las agencias turísticas promover viajes a este país, lo que provocó una caída en el número de turistas desde 2017 hasta febrero de 2018, de acuerdo con datos del Ministerio de Economía y Finanzas (MOEF, por sus siglas en inglés). En enero de 2018 se redujo la entrada de turistas chinos en 46\%, y en febrero la caída fue de 41.5\% (MOEF, 2018); de igual manera, en el mercado de la RPCh los consumidores disminuyeron sus compras en los negocios de capital surcoreano, y empresas como la cadena Lotte vieron afectadas sus ventas en dicho mercado (KBS, 2017). 
Aunado a lo anterior, las empresas de Corea del Sur han enfrentado con el paso de los años, mayor competencia (MOEF, 2018) en la región, sobre todo de las empresas de Japón y de la RPCh. Por si fuera poco, Samsung, empresa y marca emblema de los surcoreanos, presentó problemas en los aparatos electrónicos denominados smartphone Galaxy Note 7, los cuales se sobrecalentaban al ponerlos a cargar, e incluso se dieron incidentes de explosión de los mismos, situación que obligó a la empresa a retirar todos los productos que existían en el mercado, lo cual afectó la producción y el crecimiento del PIB. Para 2017 Samsung se recuperó de los problemas sufridos por dicho modelo, así que lanzó al mercado el Galaxy Note 8, y en 2018 el Galaxy Note 9; con ello mostró que se habían superado los problemas técnicos y que la empresa sigue consolidándose en el mercado, con éste y otros productos de la marca que contribuyen con su producción al crecimiento del PIB.

Para confirmar lo mencionado en el párrafo anterior, la agencia británica de asesoría empresarial, Brand Finance, determinó que la empresa Samsung Electrónica tiene el mayor valor de marca entre las empresas surcoreanas y que su valor corresponde a 88.8 billones de wones; le siguen las empresas Hyundai Motor y LG Electrónica, con un valor de marca de 11.2 billones de wones y 8 billones de wones, respectivamente. Si se contempla todo el conglomerado de empresas del Grupo Samsung, su valor asciende a 105 billones de wones, el cuarto más grande del mundo (Brand Finance, 2018; KBS, 2018).

Como se mencionó, las empresas con mayor valor se encuentran en la parte industrial de la electrónica y automotores (Brand Finance, 2018). Al analizar las principales actividades económicas que se desarrollan en Corea del Sur, vemos que éstas están relacionadas con los sectores primario, secundario y terciario. El sector primario comprende las actividades agrícolas, que representan $2.2 \%$ del PIB y generan empleos para menos de 5\% de la población trabajadora surcoreana, con el arroz como su cultivo principal, seguido por la cebada, el trigo, la soja y el sorgo; además, en dicho sector una parte de la población se dedica a la cría de ganado y a la explotación de algunos minerales, como el oro y la plata.

En lo que corresponde al sector secundario, la actividad económica se concentra en la industria, es decir en el conjunto de diversas empresas que realizan la misma actividad productiva. Por ejemplo, en Corea del Sur se tiene, en los sectores industriales, la electrónica, la petroquímica, los sectores automotriz, naval y textil. Los surcoreanos, por su dinámica económica y tecnológica, desde los años noventa han mostrado su capacidad para competir 
en la industria textil, naval, petroquímica, automotriz y electrónica, las cuales suelen representar más de $38 \%$ del PIB y generar aproximadamente el $25 \%$ del empleo de la población trabajadora surcoreana (MOEF, 2018).

Por su parte, el sector terciario, que comprende las actividades relacionadas con la industria de los servicios, del turismo, restaurantera y del comercio en Corea del Sur, en conjunto generan más de 59\% del PIB. De igual manera, en este sector se crean más de $70 \%$ de los empleos para la población trabajadora surcoreana. Es importante destacar que en el sector terciario es donde se encuentran las mayores utilidades de las actividades productivas y los menores riegos para los inversionistas, es por ello que existen los grandes almacenes, al igual que las grandes cadenas comerciales y de supermercados que dinamizan la economía de éste país.

Como se mencionó, la economía de Corea del Sur se concentra en el sector terciario, le sigue en importancia el sector secundario y con una participación relativamente baja el sector primario, todo ello como resultado de la transformación económica por la que han transitado las actividades productivas dado el compromiso del gobierno, empresas y sociedad por tener una economía sustentada en la inversión en educación y en la creación de ciencia y tecnología que les permita crecer en el PIB y mantener bajas tasas de desempleo, las cuales hasta julio de 2018 se han mantenido por debajo de 4 por ciento (MOEF, 2018).

La educación y la inversión en Investigación y Desarrollo (I\&D) son pilares que sustentan la transformación de los procesos productivos, es por ello que en Corea del Sur los montos del PIB que se invierten tanto en educación como en I\&D van a la alza: desde 2015, en educación invierten más de 5\% del PIB, y en I\&D, desde 2012, más de 4\% del PIB (MSIT, 2018; NSIT, 2018), situación que resulta positiva para las empresas y en general para la población surcoreana, pues además de permitirles incrementar la competitividad de sus empresas por las patentes que generan, y de otorgarles el derecho exclusivo de explotación, obtienen regalías por permisos que otorguen para su uso. La obtención de regalías supera al año los 7 mil millones de dólares estadounidenses; asimismo, el número de investigadores para crear ciencia y tecnología que innove los procesos y productos ha ascendido a más de 7 mil investigadores por cada millón de empleados (BM, 2018; OECD, 2018; MSIT, 2018).

En Corea del Sur la innovación en la economía y la transformación de procesos productivos están relacionadas con la capacidad para invertir más recursos financieros y 
humanos en I\&D, con lo que estimulan y desarrollan la estructura y la infraestructura necesarias que permitan a las empresas, universidades y sociedad diseñar y realizar proyectos de vanguardia acordes con las nuevas necesidades de la sociedad para consumir y producir los diversos bienes que demandan los mercados, los cuales se caracterizan por la competencia en el diseño y la creación de avances tecnológicos (BM, 2018; MOEF, 2018; MOTIE, 2018; MOE, 2018).

La competencia de los productos surcoreanos estimula el ritmo de crecimiento en el PIB, lo que permite que su economía se ubique entre las catorce con mayor valor y, con el paso de los años, alcance mayor participación en el comercio mundial por medio de sus exportaciones e importaciones; por ejemplo, en 2017 ocupó la posición cinco de los países que más exportan en el mundo, con ventas que superan los 550 mil millones de dólares, en 2016 también se ubicó en el número cinco al exportar 515 mil millones de dólares, mientras sus importaciones ascendieron, en 2017, a más de 448 mil millones, y en 2016, a 398 mil millones de dólares (OEC, 2018), lo que le da un superávit comercial en ambos años por más de 100 mil millones de dólares que contribuyen al mejoramiento de las actividades económicas y comerciales.

La consistencia en la inversión de recursos financieros del PIB en educación, así como en I\&D por parte de Corea del Sur, permite que los sectores industrial y de servicios ganen presencia en los mercados internacionales, y con ello, que las exportaciones sigan creciendo y superando las importaciones para con ello tener superávit en la balanza comercial y que los sectores relacionados con las industrias de la electrónica, semiconductores, nanotecnología, biotecnología, robótica, automotriz, petroquímica, aeroespacial, farmacéutica, energía nuclear y energía renovable, entre otras, se sigan consolidando tanto en el mercado surcoreano como en los mercados de África, Asia, América, Europa, y que las divisas internacionales de Corea del Sur superen los 300 mil millones de dólares (MOTIE, 2018; BOK, 2018; MOEF, 2018). Es importante mencionar que la industria de la construcción naval, en febrero de 2018 superó a la de la RPCh, que lideró el mercado durante 2017 (KBS, 2018).

También es importante destacar que Corea del Sur, con los montos de inversión en I\&D que superan desde 2012 el 4\% del PIB, busca tener mayor presencia en la denominada Cuarta Revolución Industrial, que se basa en los procesos de inteligencia artificial, siendo que en 
esta parte un número considerable de empresas y trabajadores surcoreanos ya tienen trazado parte del camino que deben seguir para competir y consolidar su presencia en los mercados.

Todo el trabajo desarrollado en el conjunto de la economía de Corea del Sur les condujo al diseño e implementación de la firma de diversos Acuerdos de Libre Comercio (FTA, por sus siglas en inglés) que otorgan facilidades a las empresas surcoreanas para exportar sus productos, y de igual manera que en el mercado interno existan más competidores. Es así como actualmente tienen firmados FTA con la República Popular China, Vietnam, Singapur, India, y con los países del Sudeste Asiático agrupados en la Asociación de Naciones del Sudeste Asiático (ASEAN, por sus siglas en inglés), Australia, Nueva Zelandia, Turquía, la Unión Europea, y la Asociación Europea de Libre Comercio (EFTA, por sus siglas en inglés) (MOTIE, 2018).

Las empresas surcoreanas también han incursionado en el mercado de los países de América, y tienen FTA pactados con Estados Unidos de Norteamérica, Canadá, Colombia, Perú y Chile (MOTIE, 2018). De igual manera, en el transcurso del mes de febrero de 2018 suscribió un FTA con cinco países de Centroamérica: Costa Rica, El Salvador, Honduras, Nicaragua y Panamá. Con el acuerdo se eliminarán o reducirán gradualmente los aranceles de $95 \%$ de los productos surcoreanos que se exportan al mercado de los cinco países mencionados, y con ello el MOTIE considera que se incrementarán las exportaciones, y el Instituto Coreano de Políticas Económicas Internacionales prevé un crecimiento de $0.02 \%$ en el PIB así como la creación de más de 2000 empleos, esto por la expansión del mercado para las empresas. Asimismo, como en todos los FTA que ha firmado Corea del Sur, mantiene protegidos los sectores de la agricultura relacionados con el cultivo de arroz, ajo y cebolla (KBS, 2018).

Los FTA, más la competitividad del sector industrial de Corea del Sur, contribuyen para que los resultados de las exportaciones tengan un crecimiento destacado, y en los primeros 20 días de septiembre de 2018, de acuerdo con organismos del gobierno de Corea del Sur, se tuvo un crecimiento de $21.6 \%$ en las exportaciones, las cuales se concentraron en los mercados de la RPCh, EUA, Unión Europea y Vietnam, donde destacan los productos petrolíferos, semiconductores, dispositivos de cristal líquido, barcos y automotores (KBS, 2018). 
Otro aspecto importante es que del 11 al 15 de septiembre de 2018 Corea del Sur, participó en Montevideo, Uruguay, en la primera ronda de negociaciones para un FTA con los países del Mercosur. Con las conversaciones para la firma de un acuerdo comercial, los países están concentrando diversas pláticas en lo que corresponde al comercio, inversiones, medidas sanitarias y fitosanitarias, comercio electrónico y compras gubernamentales, así como en la solución de controversias (KBS, 2018). Con ello Corea del Sur pretende consolidar su presencia en América y que sus empresas tengan más mercados para la exportación de sus productos para así estimular la creación de más empleos e impulsar un mayor crecimiento del PIB que impacte en un incremento del ingreso per cápita.

Los surcoreanos han ganado competitividad en la industria de la electrónica, semiconductores, robótica, petroquímica, automotriz, acero y naval, entre otras; pero en lo que corresponde a las empresas agrícolas relacionadas con el cultivo de arroz, ajo y cebolla, al igual que en el de la carne porcina, no tienen la capacidad para competir con los diversos socios comerciales, entre ellos los países de Centroamérica con los cuales han firmado un FTA. Dicha situación debe valorarse y analizarse más por parte de los países de América Latina, porque es en los productos agrícolas y cárnicos en los que tienen las mayores ventajas competitivas frente a Corea del Sur, y los productos industrializados de este país, con el paso de los años, ganan preferencia entre los consumidores de América, situación que genera un crecimiento en el déficit de la balanza comercial, como es el caso de México, que en los últimos periodos ha superado los 10 mil millones de dólares anuales (Secretaría de Economía, SE, 2018).

En cuanto a la expansión de los mercados por parte de las empresas de Corea del Sur, además de forzarlos para que sean más competitivos, también los presiona en el ritmo de vida, al igual que en el costo de ésta en las grandes urbes, tanto en vivienda, y esparcimiento, como en el uso de energía y los servicios necesarios para tener una vida plena.

El crecimiento económico ha permitido a la población de Corea del Sur incrementar su ingreso per cápita; y de igual manera, conforme crece el ingreso, también lo hace el costo en el nivel de vida. Dicha situación exige un mayor nivel de competencia a la sociedad y a las empresas surcoreanas, que no sólo se enfrentan a un mayor número de competidores, sino también, por el hecho de residir en un país dividido desde 1948, sufren de la tensión que suele presentarse con sus hermanos de sangre que habitan en Corea del Norte, como 
consecuencia de tener sistemas políticos y económicos opuestos, y de querer cada uno mostrar su fuerza en la península, lo cual genera incertidumbre tanto en los propios coreanos como en los países vecinos y de otras latitudes.

\section{POLÍTICA Y RELACIONES INTERCOREANAS}

Las tensiones políticas y militares entre los gobiernos de Corea del Sur y Corea del Norte han existido desde que el país se encuentra dividido; en algunos momentos han sido más álgidas que en otros. Por ejemplo, de 1950 a 1953 sufrieron la Guerra de Corea, conflicto armado que conllevó el involucramiento de otras naciones, y la tensión continuó por el deseo consistente de los norcoreanos de desarrollar un poder militar basado en misiles nucleares, con lo que provocó que naciones con amplio poder económico, político y militar se mantengan involucradas en los asuntos intercoreanos desde 1948, como es el caso de EUA, la RPCh, Japón y Rusia.

Los ensayos para probar el poder nuclear, al igual que el lanzamiento de misiles por parte de Corea del Norte durante 2016 y 2017, obligaron al gobierno de Corea del Sur a tomar la decisión de desplegar en su territorio un sistema antimisiles denominado THAAD, creado y desarrollado con tecnología militar de EUA, decisión que provocó disgusto y el rechazo por

parte del gobierno de la RPCh, que manifestó presión por la vía diplomática, política y económica en contra del gobierno surcoreano. La tensión generada entre estos dos países después de la instalación del sistema THAAD, más la situación política en Corea del Sur por escándalos de corrupción, llevaron a la destitución de la presidenta Geun Hye Park en marzo de 2017, y a que en mayo de este mismo año llegara a la presidencia Moon Jae-in (KBS, 2017).

Con su llegada al gobierno, Moon Jae-in se comprometió a normalizar la situación política al interior de Corea del Sur, así como las relaciones con el gobierno de la RPCh, con quien existía mayor irritación a causa de la instalación del sistema THAAD, por lo que en represalia, prohibió la venta de viajes turístico en grupo a Corea del Sur, limitó la distribución de contenidos culturales y artísticos, además, el grupo Lotte, que intercambió parte de sus terrenos con el gobierno surcoreano para la instalación del sistema antimisiles, tuvo que cerrar en territorio de la RPCh por la presión constante de autoridades que hacían inspecciones de higiene y seguridad, situación que alarmaba a los compradores, que dejaron de consumir en los establecimientos de este grupo empresarial surcoreano (KBS, 2017). 
Es importante mencionar que con Moon Jae-in en el poder, además de sus esfuerzos en la comunicación y mensajes a los medios tratando de reducir la tensión tanto con la RPCh como con Corea del Norte, los norcoreanos continuaron con las pruebas de lanzamiento de misiles para mostrar al mundo que tenían un desarrollo nuclear avanzado (KBS, 2018), el 3 de septiembre de 2017 realizaron su sexto ensayo nuclear en la localidad de Punggye-ri, y con ello incrementaron la tensión en la región.

Ante el crecimiento de la tensión política y militar de los gobiernos de la RPCh y Corea del Norte en contra de Corea del Sur, Moon Jae-in ha insistido en dialogar con los países vecinos, así como en reanudar la cooperación con sus hermanos de sangre de Corea del Norte; con ello deja ver su disposición para cooperar en todos los discursos oficiales, tanto dentro como fuera del país. Moon Jae-in no ha dado tregua en cuanto a tejer lazos de cooperación con los gobiernos de la RPCh y Corea del Norte, con la intención de lograr una cumbre intercoreana. Para ello, el 21 de septiembre de 2017 presentó ante la Asamblea General de las Naciones Unidas la "Iniciativa de Paz de PyeongChang", con la que pretendió lograr la participación de Corea del Norte a fin de alcanzar la paz durante los Juegos Olímpicos de Invierno de 2018 que tuvieron como sede la localidad de PyeongChang en Corea del Sur los días del 9 al 25 de febrero y tratando con ello de encontrar los puntos de coincidencia que permitan plantear soluciones al problema nuclear norcoreano (KBS, 2017; KBS, 2018).

En este contexto de diálogo y trabajo recurrente para disminuir la tensión política y económica con Corea del Norte y la RPCh, el 31 de octubre de 2017 autoridades de los gobiernos de Corea del Sur y de la RPCh acordaron normalizar sus relaciones, para ello el gobierno surcoreano se comprometió a no llevar a cabo la instalación de dispositivos adicionales del THAAD, así como a no integrar la Defensa Nacional de Misiles y no promover una alianza militar con EUA y Japón (KBS, 2017).

Los esfuerzos y el trabajo permanente que había estado desarrollando Corea del Sur para alcanzar la paz en la región se plasmaron el 1 de noviembre de 2017, cuando Moon Jaein presentó en la asamblea nacional de su país la iniciativa de cinco principios cuya intención es permitir la resolución de los problemas entre las dos Coreas. Los cinco principios consisten en: a) "Impedir choques armados, b) Desnuclearizar la península coreana, c) Solucionar con liderazgo propio los problemas intercoreanos, $d$ ) Superar pacíficamente el asunto nuclear 
norcoreano, y e) Responder con firmeza a las nuevas amenazas que pudiera presentar Corea del Norte" (KBS, 2018).

La insistencia de Corea del Sur en establecer puntos de convergencia entre las dos Coreas para lograr la paz comenzó a tener eco en el gobierno de Corea del Norte, y el 1 de enero de 2018 Jong Un Kim, líder que dirige los destinos del gobierno norcoreano, en su discurso de inicio de año planteó que enviaría a los Juegos Olímpicos de Invierno de PyeongChang una delegación de deportistas para que participaran, y de igual manera establecieran, lazos de comunicación entre las autoridades de los dos países (KBS, 2018).

Como se ha venido mencionando, el gobierno de Corea del Sur, encabezado por Moon Jae-in, desde su llegada al poder el 10 de mayo de 2017 se comprometió a realizar una búsqueda permanente de diálogo para alcanzar acuerdos que lleven la finalización de las tensiones militares en la región. El trabajo desarrollado de manera sistemática permitió que el 9 de enero de 2018 Myoung Gyon Cho, ministro de Reunificación de Corea del Sur, se reuniera con Son Gwon Ri, presidente del Comité para la Paz y la Reunificación de la Patria de Corea del Norte, en la Casa de la Paz ubicada en Panmunjeom, territorio surcoreano (KBS, 2018).

De acuerdo con el trabajo de cooperación y diálogo que se había dado entre los gobiernos y altos funcionarios de las dos Coreas, durante la inauguración y celebración de los Juegos Olímpicos de Invierno de PyeongChang, el 9 de febrero asistió una delegación de Corea del Norte de alto nivel, encabezada por Yo Jong Kim, hermana de Jong Un Kim, así como por Yong Nam Kim, presidente de la Asamblea Popular Suprema. Para el 10 de febrero Moon Jae-in, presidente de Corea del Sur, invitó a la delegación de Corea del Norte a un almuerzo, y en ese momento Yo Jong Kim hizo entrega de una carta al presidente Moon Jaein, la cual fue enviada por su hermano, en la que mostraba la disposición de su gobierno al diálogo (KBS, 2018).

Dado el acercamiento que se había dado entre los gobiernos de las dos Coreas, el 5 de marzo de 2018 el trabajo de alto nivel entre los funcionarios de Corea del Sur continuó, y en esta ocasión viajaron a Corea del Norte enviados por el presidente Moon Jae-in, el jefe de la Oficina de Seguridad Nacional Eui Yong Chung, así como el director de Inteligencia Hoon Suh. Ambos asistieron a una cena en Pyongyang, donde se reunieron con el líder norcoreano 
Jong Un $\mathrm{Kim}^{1}$ para intercambiar opiniones en favor del acercamiento entre los gobiernos, y de igual manera, para que prevalezca la confianza a fin de evitar todo tipo de confrontaciones y de establecer puntos de coincidencia con miras a celebrar una Tercera Cumbre ${ }^{2}$ Intercoreana. El 6 de marzo regresaron a Corea del Sur Eui Yong Chung y Hoon Suh, con el comunicado de que el gobierno de Jon Un Kim aceptó efectuar una Tercera Cumbre Intercoreana para finales de abril de 2018, en la Casa de la Paz de Corea del Sur (KBS, 2018).

El jefe de la Oficina de Seguridad Nacional, así como el director de Inteligencia surcoreano, después de su regreso de Corea del Norte iniciaron viajes a EUA para explicar al gobierno estadounidense los acuerdos alcanzados, de igual manera lo hicieron con los gobiernos de la RPCh, Rusia y Japón para expresar a los altos funcionarios los acuerdos, así como para pedir su colaboración, que deberá coadyuvar con la resolución de los problemas que involucran a las dos Coreas (KBS, 2018).

Con dichas visitas para informar a los gobiernos de EUA, RPCh, Rusia y Japón, más la noticia de la próxima celebración de la Tercera Cumbre Intercoreana, y por los intereses del gobierno de Corea del Norte de establecer diálogo directo con el presidente de EUA, el 9 de marzo de 2018 Donald Trump, presidente de EUA, manifestó su intención de aceptar una cumbre con el líder norcoreano Jong Un Kim y con ello iniciar los planes de trabajo tanto para la celebración de la Tercera Cumbre Intercoreana y la Primera Cumbre Corea del NorteEUA, así como para mantener informados a los gobiernos de la RPCh, Rusia y Japón (KBS, 2018).

Ya con las condiciones de diálogo, el 15 de marzo de 2018 se formó oficialmente el Comité preparatorio para la Tercera Cumbre Intercoreana, y el 29 de marzo viajó a la localidad de Tongilkak, Corea del Norte, el ministro de Reunificación de Corea del Sur, Myoung Gyon Cho, para encontrarse con Son Gwon Ri, presidente del Comité para la Paz y la Reunificación de la Patria de Corea del Norte. Ambos funcionarios indicaron en un comunicado que la Tercera Cumbre Intercoreana tendría lugar el 27 de abril de 2018, y para

${ }^{1}$ El 5 de marzo de 2018 fue la primera reunión del líder norcoreano Jong Un Kim con funcionarios de Corea del Sur desde que asumió el poder en 2012 (KBS, 2018).

${ }^{2}$ La Primera Cumbre Coreana tuvo lugar en Pyongyang, Corea del Norte, del 13 al 15 de junio del 2000. En esa ocasión se reunieron el presidente de Corea del Sur, Dae Jung Kim, con Jong Il Kim, líder supremo de Corea del Norte, teniendo como resultado una Declaración Conjunta Norte-Sur. La Segunda Cumbre también se celebró en Pyongyang del 2 al 4 de octubre del año 2007; en ésta se reunieron Jong Il Kim y el presidente surcoreano Moo Hyun Roh; el resultado fue una Declaración Conjunta de Paz y Prosperidad (KBS, 2018). 
continuar con el acercamiento entre los dos países, a finales de marzo artistas surcoreanos viajaron a Pyongyang para ofrecer diversas actuaciones los días 1 y 3 de abril. Asistieron a dichos eventos el líder norcoreano Jong-Un Kim y su esposa Sol Ju Ri (KBS, 2018).

Para reducir la tensión entre las dos Coreas se realizó un diálogo y encuentros continuos de alto nivel entre los funcionarios de los dos países, y el 20 de abril de 2018, en reunión plenaria del Comité Central del Partido de los Trabajadores encabezado por Jong Un Kim, se declaró el final de la política sustentada en el desarrollo nuclear y el progreso económico en sintonía. De igual manera, Corea del Norte manifestó su compromiso de no efectuar ensayos nucleares, y planteó que cerraría el centro nuclear ubicado en Punggye-ri, condado de Kilju de la provincia Hamgyong del Norte; asimismo, indicó que sus armas nucleares y convencionales no apuntarán hacia territorio surcoreano. Con dicho pronunciamiento se allanó el camino para que el 27 de abril el presidente de Corea del Sur, y el líder de Corea del Norte, celebraran la Tercera Cumbre Intercoreana en la Aldea de la Tregua de Panmunjeom, siendo ésta la primera que tiene lugar en Corea del Sur. Ambos firmaron la Declaración de Panmunjeom $2018^{3}$ (KBS, 2018).

En el marco de la Declaración de Panmunjeom, entre otros acuerdos establecieron organizar reuniones de las familias separadas, en el mes de agosto; también consideraron relevante la instalación, en el complejo industrial de Gaesong, de una oficina conjunta de enlace intercoreana que les permita garantizar el intercambio y la cooperación con el sector privado. De igual manera se comprometieron a dar continuidad a los trabajos de la Tercera Cumbre Intercoreana en los próximos meses de 2018, la cual tendría como sede Pyongyang, Corea del Norte (KBS, 2018).

En lo que respecta a los acuerdos para que las familias separadas de las dos Coreas puedan reencontrarse, ambos gobiernos se organizaron para que en agosto aproximadamente 70 familias tuvieran algunas horas de convivencia familiar, así como grupal. Por la demanda que existe, de 56 mil personas, de las cuales $85 \%$ tienen más de setenta años, altos funcionarios de la Cruz Roja de Corea del Sur y Corea del Norte han estado detallando y

${ }^{3}$ Declaración de Panmunjeom para la paz, prosperidad y reunificación de la península coreana, firmada por el presidente de la República de Corea, Moon Jae In, y el presidente de la Comisión de Asuntos Estatales de la República Popular Democrática de Corea, Kim Jong Un. Celebraron una cumbre en la Casa de la Paz de Panmunjeom el 27 de abril de 2018 (KBS, 2018). Para mayor información de los acuerdos consúltese: http://world.kbs.co.kr/special/northkorea/contents/archives/summit/summit_2018.htm?lang=s\#summitResult 
analizando un calendario para próximas reuniones, considerando posibles nuevas fechas en los últimos días de octubre de 2018. De igual manera están diseñando estrategias mediante Tecnologías de Información y Comunicación, para que por medio de videoconferencias se tengan reencuentros y la posibilidad de que las familias puedan encontrarse en sus pueblos natales y no en el complejo turístico del monte Kumgang de Corea del Norte (KBS, 2018).

Es importante destacar que antes y después de la Tercera Cumbre Intercoreana organizada en Panmunjeom el 27 de abril de 2018. El presidente de Corea del Sur y altos funcionarios de su gobierno estuvieron trabajando y comunicando al gobierno de EUA los puntos a tratar con Jong Un Kim, así como el interés del gobierno norcoreano de tener una reunión de alto nivel con el presidente Donald Trump. El trabajo desarrollado entre los funcionarios de Corea del Sur y de EUA permitió, el 12 de junio de 2018, un encuentro en el Hotel Capella de la isla de Sentosa en Singapur, siendo ésta la primera reunión que se tiene entre un presidente estadounidense y un líder norcoreano. Para dicho encuentro contribuyeron, además de los países mencionados, la RPCh, que facilitó aviones para el desplazamiento de la comitiva norcoreana, y Rusia, con aviones de carga (CNN, 2018; KBS, 2018).

Como se mencionó, el 12 de junio de 2018, por primera vez en la historia se reunieron un presidente estadounidense y un líder norcoreano, y los acuerdos que firmaron se concentran en cuatro puntos: el primero señala que EUA y Corea del Norte establecerán nuevas relaciones en pro de la paz y la prosperidad. En el segundo se comprometen a esforzarse para lograr una paz permanente y estable en la península coreana. En el tercero confirman la Declaración de Panmunjeom que fue adoptada el 27 de abril de 2018; y en el cuarto punto se comprometen a buscar y a recuperar los cadáveres de prisioneros y desaparecidos durante la Guerra de Corea que tuvo lugar de 1950 a 1953. Al finalizar la reunión, ambos líderes manifestaron su deseo de superar las décadas de tensión y antagonismo; para ello, funcionarios de alto nivel seguirán manteniendo reuniones que les permitan afianzar la cooperación y la paz en la región (KBS, 2018).

Con los acuerdos anteriores se presenta también un trabajo de mayor cooperación y diálogo que permite ir construyendo las bases para la paz en la región. De este modo, en agosto de 2018 fueron autorizadas más reuniones de familias separadas, y el 14 de septiembre se inauguró la oficina de enlace intercoreana en el Complejo Industrial de Gaesong, con lo 
que se facilitó la comunicación intercoreana, así como los intercambios civiles y el traspaso fronterizo durante todo el año. También es importante destacar que en la Declaración de Panmunjeom los líderes se comprometieron a dar continuidad a la misma en territorio de Corea del Norte, y los días 18 al 20 de septiembre el presidente Moon Jae-in y su esposa Jung Sook Kim, más una comitiva de altos funcionarios, visitaron Corea del Norte a fin de concretar los acuerdos para impulsar la paz y la cooperación. A su arribo fueron recibidos en el aeropuerto Sunan de Pyongyang por Jong Un Kim y su esposa Sol Ju Ri, situación que favorece la confianza para alcanzar la paz en favor de la sociedad de los dos países (KBS, 2018).

Luego de varias reuniones de trabajo entre Moon Jae-in y Jong Un Kim, el 19 de septiembre anunciaron la "Declaración Conjunta de Pyongyang", en la cual muestran compromisos para reducir la tensión militar por medio del cese de los ejercicios militares entre EUA y Corea del Sur, así como el cierre definitivo del centro de pruebas de misiles de Corea del Norte que se encuentra establecido en Dongchang-ri, el desmantelamiento de las instalaciones nucleares de Yongbyon, y la realización de una visita a la capital surcoreana por parte del líder norcoreano Jong Un Kim. Otros aspectos importantes tienen que ver con la parte económica, en la que se comprometen a iniciar en 2018 las obras para la conexión de la red ferroviaria intercoreana y normalizar el turismo en el monte Geumgang de Corea del Norte; de igual manera, trabajar conjuntamente para organizar los Juegos Olímpicos de Verano de 2032, y celebrar en conjunto el centenario del Grito de Independencia del 1 de marzo de 2019 (KBS, 2018).

El diálogo entre los gobiernos seguirá, y se espera que la cooperación mostrada hasta el mes de septiembre de 2018 entre los líderes de las dos Coreas, y los mandatarios de EUA, RPCh, Rusia y Japón permita consolidar la paz, y que el progreso económico lleve a Corea del Sur y a Corea del Norte a la reunificación en un tiempo no muy lejano.

\section{REFLEXIONES FINALES}

Durante 2018 Corea del Sur mostró nuevamente al mundo su fortaleza económica sustentada en la producción de productos con un alto contenido tecnológico que les permite realizar más exportaciones que importaciones. Sobresalen las ventas de productos petrolíferos, semiconductores, pantallas de cristal líquido, embarcaciones y automotores. 
Por el nivel de producción y la innovación de procesos, al igual que de productos, entre las empresas surcoreanas que están posicionadas tanto en el mercado doméstico como en el internacional se encuentran Samsung Electrónica, Hyundai Motor y LG Electrónica, las cuales, para continuar sus procesos tecnológicos de vanguardia, invierten recursos con el fin de tener presencia en universidades y allegarse capital humano altamente calificado que les permita seguir innovando y ganar presencia en los avances tecnológicos.

La penetración y consolidación de los productos de alta tecnología surcoreanos en mercados del continente asiático, americano, europeo y africano, contribuyen con una política económica y comercial de apertura, la cual se ha caracterizado por la firma de FTA con países que tienen las más grandes economías del planeta, entre ellos EUA y la RPCh. De igual manera, dada la magnitud de sus niveles de producción de sus empresas, durante 2018 firmaron un FTA con países de Centroamérica, e iniciaron negociaciones para firmar con países del Mercosur, en un intento por consolidarse en todos los mercados y de impulsar el crecimiento de su PIB por medio de un crecimiento en las exportaciones.

Corea del Sur, a pesar de haber alcanzado altos niveles de competitividad en sus sectores industriales, en todas sus negociaciones comerciales siempre cuida y protege al sector agrícola, principalmente a los productores de arroz, soja y cebolla, entre otros, porque dichos sectores tienen un nivel de competitividad bajo en comparación con los productores de Centroamérica y Sudamérica. Ante dicha situación, en sus acuerdos comerciales Corea del Sur ha sabido diseñar estrategias que le permiten ganar-ganar, tanto en el mercado exterior como en el doméstico.

La situación económica de Corea del Sur, la mayor parte del tiempo está acompañada de la tensión política y militar que vive con sus vecinos y hermanos de sangre de Corea del Norte; dicha situación, resultado de ensayos nucleares y de pruebas de misiles que realizan los norcoreanos en respuesta a los ejercicios militares que realizan las fuerzas de EUA y Corea del Sur.

Para tratar de eliminar esa tensión, desde principios de 2018 los gobiernos de las dos Coreas participaron de manera conjunta en los Juegos Olímpicos de Invierno que tuvieron lugar en PyeongChang, Corea del Sur. De igual manera, el 27 de abril los líderes de Corea del Sur, Moon Jae-in, y de Corea del Norte, Jong Un Kim, celebraron la Tercera Cumbre Intercoreana en Panmunjeom, territorio surcoreano, donde acordaron y firmaron acuerdos 
que permiten el reencuentro de familias separadas y, de igual manera, trabajar por la paz en la península.

El trabajo desarrollado entre los gobiernos de las dos Coreas, más el diálogo que mantienen con los gobiernos de EUA, RPCh, Rusia y Japón, contribuyeron para que el 12 de junio se realizara en Singapur la primera reunión entre un presidente de EUA y un líder de Corea del Norte, los cuales iniciaron un diálogo que los llevaría a afianzar la cooperación y la paz en la región.

Los trabajos de cooperación y el diálogo permanente continúan entre los gobiernos de las dos Coreas, y durante el mes de septiembre tuvieron la segunda sesión de la Tercera Cumbre Intercoreana, pero ahora en Pyongyang, capital de Corea del Norte. El líder del país anfitrión se comprometió a destruir las plantas nucleares y de producción de misiles; por su parte, el presidente de Corea del Sur inició una serie de trabajos para la construcción de vías férreas que permitan la conexión de trenes entre el Sur y el Norte e impulsen la economía norcoreana por medio del Complejo Industrial de Gaesong. Pero sobre todo, para mantener una comunicación que evite confrontaciones, así como la tensión militar, en la región, y a la vez genere condiciones para que la economía crezca y logre un mayor bienestar para la población de la península coreana.

\section{REFERENCIAS BIBLIOGRÁFICAS}

Banco Mundial BM (2018), Ciencia y Tecnología, 20 de agosto. Recuperado de: https://datos.bancomundial.org/indicador/SP.POP.SCIE.RD.P6?view=chart.

Banco Mundial (2018), "Crecimiento del PIB (\% anual)", 30 de agosto. Recuperado de: https://datos.bancomundial.org/indicador/NY.GDP.MKTP.KD.ZG?view=chart.

Banco Mundial (2018), Exportaciones de productos de alta tecnología (\% de las exportaciones de productos manufacturados), 28 de agosto. Recuperado de: https://datos.bancomundial.org/indicador/TX.VAL.TECH.MF.ZS?view=chart.

Banco Mundial (2018), Cargos por el uso de propiedad intelectual, recibos (balanza de pagos US\$ a precios actuales, 28 de agosto. Recuperado de: https://datos.bancomundial.org/indicador/BX.GSR.ROYL.CD?view=chart. 
Banco Mundial (2018), PIB per cápita (US\$ a precios actuales), 7 de septiembre. Recuperado de: https://datos.bancomundial.org/indicador/NY.GDP.PCAP.CD?view=chart.

BOK (2018), The Bank of Korea, Principal Indicators, 31 de agosto. Recuperado de: http://www.bok.or.kr/eng/main/main.do.

BOK (2018), The Bank of Korea, International Reserves and External Debts, 16 de septiembre. Recuperado de: http://www.bok.or.kr/eng/main/contents.do?menuNo=400196.

Brand Finance (2018), "Valuation Reports", 10 de septiembre. Recuperado de: http://brandfinance.com/knowledge-centre/reports/.

Brand Finance (2018), South Korea 2018, The Annual Report on the Most Valuable South Korea brands, agosto, 10 de septiembre. Recuperado de: http://brandfinance.com/images/upload/brand_finance_south_korea_50_2018_locked .pdf.

CNN (2018), Lo que dice el apretón de manos de Kim y Trump (y en general su encuentro), según los expertos, 12 de junio. Publicado y recuperado de: https://cnnespanol.cnn.com/2018/06/12/lo-que-dice-el-apreton-de-manos-de-kim-ytrump-y-en-general-su-encuentro-segun-los-expertos/.

KBS (2017), El sector servicios coreano registra el mayor déficit de su historia, 5 de septiembre. Recuperado de: http://world.kbs.co.kr/service/news_view.htm?lang=s\&Seq_Code=57060.

KBS (2018), Corea del Sur suscribe un TLC con 5 naciones centroamericanas, 21 de febrero. Publicado y recuperado de: http://world.kbs.co.kr/spanish/news/news_Ec_detail.htm?No=59881\&id=Ec.

KBS (2018), Corea del Sur negocia un acuerdo comercial con Mercosur, 10 de septiembre. Publicado y recuperado de: http://world.kbs.co.kr/service/news_view.htm?lang=s\&Seq_Code=62746.

KBS (2018), Samsung es la empresa surcoreana con el mayor valor de marca, 10 de septiembre. Publicado y recuperado de: http://world.kbs.co.kr/service/news_view.htm?lang=s\&Seq_Code=62739. 
KBS (2018), Corea supera a China en la industria de construcción naval, 20 de marzo. Publicado y recuperado de: http://world.kbs.co.kr/spanish/news/news_Ec_detail.htm?lang=s\&id=Ec\&No=60084 \&current_page $=$.

KBS (2018), La balanza turística registró en 2017 el peor déficit en la historia, 10 de marzo. Publicado y recuperado de: http://world.kbs.co.kr/spanish/news/news_Ec_detail.htm?No=59873\&id=Ec.

KBS (2018), Las exportaciones continúan al alza en septiembre, 21 de septiembre. Publicado y recuperado de: http://world.kbs.co.kr/service/news_view.htm?lang=s\&Seq_Code=62919.

KBS (2018), Park Geun Hye es removida y enjuiciada por corrupción, Publicado el 10 de marzo, y recuperado el 16 de septiembre de 2018, de: http://world.kbs.co.kr/service/archive_view.htm?lang=s\&id=sub_index\&board_seq= 932.

KBS (2018), Conflicto y reconciliación entre Seúl y Beijing por el THAAD, publicado el 31 de octubre, y recuperado el 30 de agosto de 2018, de: http://world.kbs.co.kr/service/archive_view.htm?lang=s\&id=sub_index\&board_seq= 942.

KBS (2018), Cumbre intercoreana 2018, 28 de abril. Publicado y recuperado de: http://world.kbs.co.kr/special/northkorea/contents/archives/summit/summit_2018.htm ?lang=s\#summitResult.

KBS (2018), La delegación norcoreana regresa a Pyongyang tras su visita a Corea del Sur, publicado el 27 de febrero, y recuperado el 28 de febrero, de: http://world.kbs.co.kr/spanish/news/news_IK_detail.htm?No=59966\&id=IK.

KBS (2018), El enviado del Moon Jae In viaja a Corea del Norte, publicado el 5 de marzo, y recuperado el 7 de marzo, de: http://world.kbs.co.kr/spanish/news/news_IK_detail.htm?No=60048\&id=IK. 
KBS (2018), La delegación surcoreana se reúne con Kim Jong Un, publicado el 5 de marzo, y recuperado el 7 de marzo, de: http://world.kbs.co.kr/spanish/news/news_IK_detail.htm?No=60050\&id=IK.

KBS (2018), La prensa del Norte informa de la reunión entre Kim Jong Un y la delegación surcoreana, publicado el 6 de marzo, y recuperado el 7 de marzo, de: http://world.kbs.co.kr/spanish/news/news_IK_detail.htm?No=60061\&id=IK.

KBS (2018), Corea del Sur y Corea del Norte acuerdan celebrar una cumbre bilateral en abril, publicado el 6 de marzo, y recuperado el 10 de marzo, de: http://world.kbs.co.kr/service/news_view.htm?lang=s\&Seq_Code=60069.

KBS (2018), Regresa a Seúl el enviado especial de Moon Jae In tras visitar Pyeongyang, publicado el 6 de marzo, y recuperado el 7 de marzo, de http://world.kbs.co.kr/spanish/news/news_IK_detail.htm?No=60068\&id=IK.

KBS (2018), Proceso de Concreción de la I Reunión Cumbre Intercoreana, publicado en junio de 2000, y recuperado el 30 de abril de 2018, de: http://world.kbs.co.kr/special/northkorea/contents/archives/summit/summit_2000.htm ?lang $=\mathrm{s}$.

KBS (2018), Cumbre Intercoreana 2007. Etapa de preparación, publicado en octubre de 2007 , y recuperado el 30 de abril de 2018, de: http://world.kbs.co.kr/special/northkorea/contents/archives/summit/summit_2007.htm ?lang $=\mathrm{s}$.

KBS (2018), Declaración de Panmunjeom de la Cumbre Intercoreana 2018, 28 de abril. Publicado $\mathrm{y}$ recuperado de: http://world.kbs.co.kr/special/northkorea/contents/archives/summit/summit_2018.htm ?lang=s\#summitResult.

KBS (2018), Segundo día del reencuentro de familias separadas de las dos Coreas, 25 de agosto. Publicado y recuperado de: http://world.kbs.co.kr/service/news_view.htm?lang=s\&Seq_Code=62522. 
KBS (2018), Las dos Coreas impulsarán nuevos reencuentros de familias separadas en 2018, 27 de agosto. Publicado y recuperado de: http://world.kbs.co.kr/service/news_view.htm?lang=s\&Seq_Code=62539.

KBS (2018), Revelan el contenido del acuerdo firmado entre EUA y Corea del Norte, 12 de junio. Publicado y recuperado de http://world.kbs.co.kr/service/news_view.htm?lang=s\&Seq_Code=61464.

KBS (2018), Uno de los aviones facilitados por China para la cumbre de Singapur llega a Beijing, 13 de junio. Publicado y recuperado de: http://world.kbs.co.kr/spanish/news/news_In_detail.htm?lang=s\&id=In\&No=61467\& current_page $=$.

KBS (2018), Rusia aboga por desactivar las sanciones contra Corea del Norte, 14 de junio. Publicado y recuperado de: http://world.kbs.co.kr/service/news_view.htm?lang=s\&Seq_Code=61494.

KBS (2018), La oficina intercoreana de enlace será inaugurada el día 14, 12 de septiembre. Publicado y recuperado de: http://world.kbs.co.kr/service/news_view.htm?lang=s\&Seq_Code=62778.

KBS (2018), Trump reitera que suspenderá las maniobras militares conjuntas con Seúl, 13 de junio. Publicado y recuperado de: http://world.kbs.co.kr/spanish/news/news_In_detail.htm?lang=s\&id=In\&No=61469\& current_page $=$.

KBS (2018), Kim y Trump apuestan por una “desnuclearización completa”, 13 de junio Publicado $\mathrm{y}$ recuperado de: http://world.kbs.co.kr/spanish/news/news_In_detail.htm?lang=s\&id=In\&No=61475\& current_page $=$.

KBS (2018), La prensa norcoreana cubre la Declaración Conjunta de Pyongyang, 20 de septiembre. Publicado y recuperado de: http://world.kbs.co.kr/service/news_view.htm?lang=s\&Seq_Code=62895. 
KBS (2018), Lideres de las dos Coreas adoptan la Declaración Conjunta de Pyongyang de septiembre, 19 de septiembre. Publicado y recuperado de: http://world.kbs.co.kr/service/news_view.htm?lang=s\&Seq_Code=62885.

KBS (2018), Pyongyang cerrará sus instalaciones nucleares, 19 de septiembre. Publicado y recuperado de: http://world.kbs.co.kr/service/news_view.htm?lang=s\&Seq_Code=62883.

KBS (2018), Pyongyang recibe con gran euforia a Moon Jae In, 18 de septiembre. Publicado y recuperado de: http://world.kbs.co.kr/service/news_view.htm?lang=s\&Seq_Code=62862.

KBS (2018), Los líderes de las dos Coreas visitan el monte Baekdu, 20 de septiembre. Publicado y recuperado de: http://world.kbs.co.kr/service/news_view.htm?lang=s\&Seq_Code=62894.

KBS (2018), Moon se dirige en persona a la ciudadanía norcoreana, 20 de septiembre. Publicado $\mathrm{y}$ recuperado de: http://world.kbs.co.kr/service/news_view.htm?lang=s\&Seq_Code=62891.

Lee, Yoonseo (2018), Corea del Sur ocupó el quinto lugar entre los países de la OCDE, 31 de mayo. Publicado y recuperado de http://spanish.korea.net/NewsFocus/Business/view?articleId=159624.

Ministry of Education (MOE) (2018), Education for the future, 5 de septiembre. Recuperado de: http://english.moe.go.kr/main.do?s=english.

MOE (2018), History, 30 de agosto. Recuperado de: http://english.moe.go.kr/sub/info.do?m=0104\&page=0104\&s=english.

MOEF (2018), Ministry of Economy and Finance, 31 de agosto. Recuperado de http://english.moef.go.kr/di/selectTbDailyIndDtl.do.

MOEF (2018), Ministry of Economy and Finance, Economic Bulletin, agosto, 2018, 15 de septiembre. Recuperado de: http://english.moef.go.kr/pu/selectTbPublicDtl.do?boardCd=P0002\&seq=. 
MOTIE (2018), Ministry of Trade, Industry and Energy, Trade Balance, 7 de septiembre. Recuperado de: http://english.motie.go.kr/www/main.do.

MOTIE (2018), Korea's FTA Network, 16 de septiembre. Recuperado de: http://english.motie.go.kr/en/if/ftanetwork/ftanetwork.jsp.

MSIT (2018), Ministry of Science, ICT and Future Planning, 7 de septiembre. Recuperado de: http://english.msip.go.kr/english/main/main.do.

MSIT (2018), The Republic of Korea Rides on the Big Road of the $4^{\text {th }}$ Industrial Revolution, 5 de septiembre. Recuperado de: http://english.msip.go.kr/english/main/main.do.

MSIT (2018), Master Plan for the Intelligent Information Society, 20 de julio. Recuperado de: http://english.msip.go.kr/english/msipContents/contents.do?mId=NDYx.

MSIT (2018), Laws in Science and Technology, 20 de julio. Recuperado de: http://english.msip.go.kr/english/msipContents/contents.do?mId=NDcy.

National Science \& Technology Information (NSTI) (2018), Statistics, 2 de septiembre. Recuperado de: http://www.ntis.go.kr/en/GpIntroduction.do.

NSTI (2018), Statistics, 2 de septiembre. Recuperado de: http://www.ntis.go.kr/en/GpExpenditureTotal.do.

NSTI (2018), R\&D Expenditure (Organization), 2 de septiembre. Recuperado de: http://www.ntis.go.kr/en/GpInstitutional.do.

NSTI (2018), Researcher (Total), 2 de septiembre. Recuperado de: http://www.ntis.go.kr/en/GpResearcherTotal.do.

NSTI (2018), Researcher (Organization), 2 de septiembre. Recuperado de: http://www.ntis.go.kr/en/GpResearchInstitutional.do.

OECD Factbook, Economic (2018), Environment and Social Statistics 2015-2016, 25 de agosto. Recuperado de: http://www.oecd.org/publications/oecd-factbook18147364.htm.

OECD (2018), OECD Economic Surveys Korea, junio, Overview, 10 de septiembre. Recuperado de: http://www.oecd.org/eco/surveys/Korea-2018-OECD-economicsurvey-overview.pdf. 
OEC (2018), Corea del Sur, exportaciones e importaciones, 12 de septiembre, Recuperado de: https://atlas.media.mit.edu/es/profile/country/kor/.

SE (2018), Secretaría de Economía, Acciones y Programas, Comercio Exterior, 16 de septiembre. Recuperado de: http://www.economiasnci.gob.mx/sic_php/pages/estadisticas/.

Yonhap (2018), La OCDE mantiene la previsión de crecimiento económico de Corea del Sur para 2018 en 3\%, 30 de mayo. Recuperado de: http://spanish.yonhapnews.co.kr/national/2018/05/30/0300000000ASP201805300025 00883.HTML. 